\title{
Bronchoscopic Diagnosis and Treatment of Primary Tracheobronchial Amyloidosis: A Retrospective Analysis from China
}

\author{
Xiaoxiao Lu, ${ }^{1}$ Bixiu He, ${ }^{1}$ Ge Wang, ${ }^{2}$ Baimei He, ${ }^{1}$ Lijing Wang, ${ }^{1}$ and Qiong Chen ${ }^{1}$ \\ ${ }^{1}$ Department of Geriatrics, Respiratory Medicine, Xiangya Hospital, Central South University, Changsha, Hunan 410008, China \\ ${ }^{2}$ Department of Information, Zhengzhou Maternal and Children Health Hospital, Zhengzhou, Henan 450012, China \\ Correspondence should be addressed to Lijing Wang; wanglijing33@163.com
}

Received 3 November 2016; Accepted 28 December 2016; Published 19 January 2017

Academic Editor: Mohamad A. Hussein

Copyright (C) 2017 Xiaoxiao Lu et al. This is an open access article distributed under the Creative Commons Attribution License, which permits unrestricted use, distribution, and reproduction in any medium, provided the original work is properly cited.

\begin{abstract}
Objective. To assess the value of bronchoscopy in the diagnosis and treatment of primary tracheobronchial amyloidosis (TBA), in order to reduce misdiagnosis rates and improve prognosis. Methods. Clinical data of 107 patients with TBA reported from 1981 to 2015 in China were retrospectively analyzed for clinical features, bronchoscopic manifestations, pathologies, treatments, and outcomes. Results. 105 of 107 TBA patients were pathologically confirmed by bronchoscopy. Main bronchoscopic manifestations of TBA were single or multiple nodules and masses within tracheobronchial lumens; local or diffuse luminal stenosis and obstruction; luminal wall thickening and rigidity; rough or uneven inner luminal walls; congestion and edema of mucosa, which was friable and prone to bleeding upon touch; and so forth. 53 patients were treated with bronchoscopic interventions, like Nd-YAG laser, highfrequency electrotome cautery, freezing, resection, clamping, argon plasma coagulation (APC), microwaving, stent implantation, drug spraying, and other treatments. 51 patients improved, 1 patient worsened, and 1 died. Conclusion. Bronchoscopic biopsy is the primary means of diagnosing TBA. A variety of bronchoscopic interventions have good short-term effects on TBA. Bronchoscopy has important value in the diagnosis, severity assessment, treatment, efficacy evaluation, and prognosis of TBA.
\end{abstract}

\section{Introduction}

Primary tracheobronchial amyloidosis (TBA) refers to a group of rare diseases with varied clinical manifestations which are caused by abnormal deposition of $\beta$-sheet amyloids in the tracheal and bronchial submucosa in the absence of systemic amyloidosis. Incidence of amyloidosis is about $8 / 1$ million [1]. TBA accounts for $1.1 \%$ of all amyloidoses [2]. In China, only over 200 cases of TBA have been reported so far. The disease lacks typical clinical symptoms and imaging manifestations [3], so if clinicians do not have sufficient knowledge about TBA, misdiagnosis and missed diagnosis will be highly likely [4]. In recent years, with the popularization of bronchoscopy, the number of TBA cases diagnosed by bronchoscopy has significantly increased than before. The rapid development of bronchoscopic interventional techniques has provided TBA patients with new treatment options. This paper aims to retrospectively analyze
2 cases of TBA patients in our hospital in recent years as well as 107 TBA patients reported from 1981 to 2015 in China, with the focus on explaining the value of bronchoscopy in the diagnosis and treatment of TBA.

\section{Subjects and Methods}

2.1. Retrieval Methods. With "primary tracheobronchial amyloidosis" as search term, CNKI, VIP, and Wanfang Databases were retrieved. Meanwhile, PubMed database was searched with "primary tracheobronchial amyloidosis" and "China" as search terms. Data collected included age, gender, disease duration, clinical manifestations, presence or absence of misdiagnosis, imaging manifestations, bronchoscopic manifestations, pathology, treatments, and prognosis.

2.2. Screening Methods. Inclusion criteria were (1) patients histopathologically diagnosed with TBA and (2) those with 

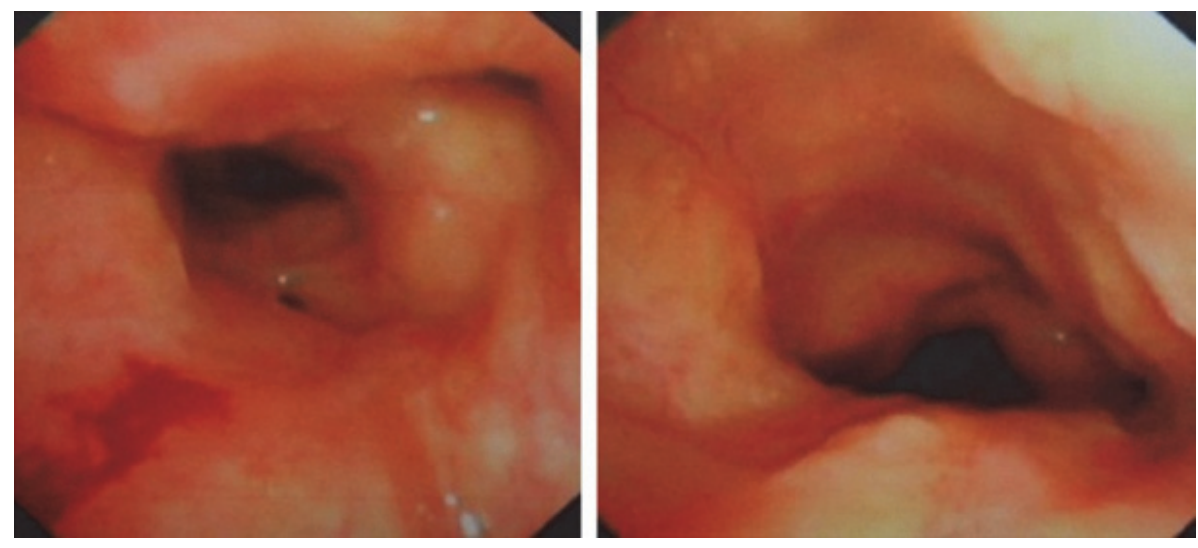

FIGURE 1: Electronic bronchoscopy photographs of the first patient. Pictures above reveal significant swelling and hypertrophy of the tracheal and bronchial mucosa.

complete clinical data. Exclusion criteria were (1) duplicate reports; (2) secondary tracheobronchial amyloidosis or systemic amyloidosis; and (3) patients with accompanying pharyngolaryngeal or pulmonary parenchymal amyloidosis. According to the inclusion and exclusion criteria, we screened out 54 papers [5-28] from the four databases to enroll 107 TBA patients, which totaled 109 cases after adding the 2 cases in our hospital.

2.3. Data Analysis Methods. Data were analyzed using SPSS 17.0 statistical software. Normally distributed measurement data were expressed as mean $\pm \mathrm{SD}$; abnormally distributed measurement data were expressed as median, while enumeration data were presented as constituent ratio.

\section{Case Information}

3.1. Case 1. The patient is a 68-year-old male who complained of recurrent cough, expectoration, and progressive dyspnea for more than 30 years. He was admitted to our hospital after being diagnosed with chronic bronchitis, bronchiectasis, and endobronchial tuberculosis in other hospitals and treated with antibiotics numerous times. Despite treatment, his symptoms progressively worsened. In 1988, the patient was managed with a combination of isoniazid, streptomycin, and rifampicin for a diagnosis of endobronchial tuberculosis after bronchoscopy in another hospital. Three months later, with no improvement on these antitubercular agents and seeking a second opinion and treatment, the patient discontinued the treatment and came to our hospital.

The patient presented with diminished breath sounds and slight bibasilar wheezing rales and crackles. Routine blood test was normal. A PPD test was negative. Sputum samples were negative for bacteria, acid-fast bacilli, and fungi. A recent chest CT demonstrated extensive thickening of the walls of the trachea and bronchi at different levels with luminal narrowing. Electronic bronchoscopy revealed significant swelling and hypertrophy of the tracheal and bronchial mucosa (Figure 1). Pathological examination of the endobronchial biopsy specimen revealed chronic inflammation of the mucosa with amyloid deposition, PAS stain
$(-)$, digestive PAS stain (-), and Congo red stain (+). There was no evidence of extrapulmonary organ involvement in amyloidosis. Based on the above comprehensive evaluation, the diagnosis of primary TBA was established.

3.2. Case 2. A 53-year-old male patient was admitted because of recurrent cough, expectoration, bloody sputum for 20 years, and shortness of breath for 5 years. He had been repeatedly diagnosed with bronchiectasis and chronic bronchitis in other hospitals. Physical examination on admission revealed moist rales in bilateral lower lungs. Routine blood test was normal. Tuberculosis antibody was negative. $G$ test was negative. Chest CT showed thickening and calcification of main bronchial wall as well as left and right bronchial walls and secondary atelectasis of right middle lobe. Electronic bronchoscopy findings were mucosal hypertrophy, swelling, significant hyperemia, unevenness and luminal stenosis which were visible in the trachea, left and right main bronchi, right upper lobe and right intermediate bronchi, right lower lobe bronchus, as well as left upper and lower lobe bronchi, and total occlusion of right middle lobe bronchus (Figure 2). Pathological examination results showed chronic mucosal inflammation and amyloid deposition, acid-fast staining (-), digested PAS staining (-), and Congo red staining (+). No evidence of amyloidosis was observed in other parts. The patient was diagnosed with primary TBA, who was treated with melphalan $10 \mathrm{mg}$ Qd, methylprednisolone $40 \mathrm{mg}$ Qd, and bronchoscopic Nd-YAG laser twice to get markedly relieved symptoms. His condition was stable during a 6month follow-up.

\section{Results}

4.1. General Information. Among 109 patients included, 23 cases were reported before the year 2000 , while 86 cases were reported after 2000. These patients consisted of 67 males and 42 females, with a male/female ratio $=1.6: 1$. Age of onset ranged between 18 and 81 years, with a mean of $(52.16 \pm$ 11.33) years. As for disease duration, the longest was 30 years, whereas the shortest was 12 hours, with a median of 2 years. Main clinical manifestations were cough, shortness 

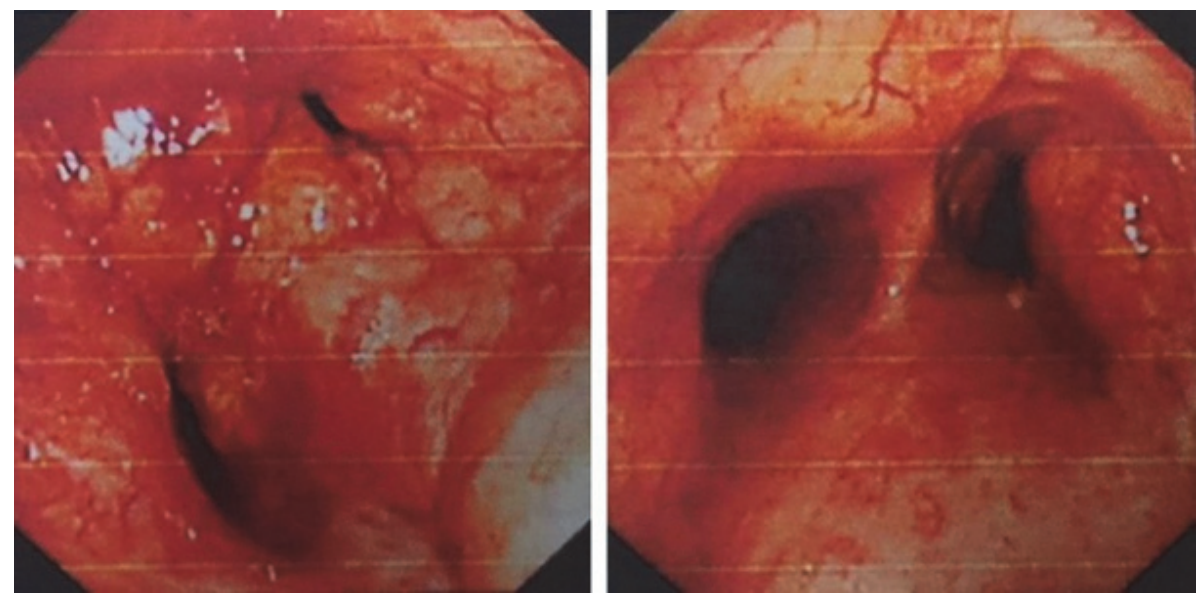

FIGURE 2: Electronic bronchoscopy photographs of the second patient. Pictures above reveal mucosal hypertrophy, swelling, significant hyperemia, unevenness and luminal stenosis in the trachea and bronchi, and total occlusion of right middle lobe bronchus.

of breath or progressive dyspnea, expectoration, hemoptysis or bloody sputum, fever, chest tightness, hoarseness, and so forth. Misdiagnosis rate at first hospitalization was up to $45 \%$. Most patients were misdiagnosed as chronic bronchitis, endobronchial tuberculosis, lung tumor, bronchial asthma, pulmonary infections, and so forth (see Table 1 for details).

4.2. Imaging Manifestations. 82 patients underwent lung CT examination, who were manifested mainly with tracheal stenosis, tracheal wall thickening, calcification of wall, patchy shadows, atelectasis, and hilar space-occupying lesions. 59 patients underwent chest X-ray examination, who were manifested often with increased and disorderly lung markings, atelectasis, patchy shadows, bronchitis, emphysema, luminal stenosis, and enlarged hilar shadows (see Table 2 for details).

4.3. Bronchoscopic and Histopathological Examinations. 107 patients underwent bronchoscopy. Main manifestations included multiple nodules or masses within tracheal and bronchial lumens, luminal stenosis or occlusion, bronchial wall thickening, brittle and easily bleeding mucosa, mucosal roughness or unevenness, mucosal hyperemia and edema, pale mucosa, and wall rigidity. Histopathology of all patients showed amyloid deposition in tracheal and bronchial submucosa. Among them, brick red amyloid was reported in 50 cases by Congo red staining, while only 22 cases mentioned observation of yellow-green birefringence of amyloid under polarizing microscope (see Table 3 for details).

4.4. Treatments and Outcomes. 53 patients were treated with bronchoscopic interventions, including Nd-YAG laser, argon plasma coagulation (APC), freezing, drug spraying, clamping, resection, high-frequency electrotome cautery, stent implantation, and microwaving. Among these patients, 20 received bronchoscopic therapy alone; 32 received bronchoscopic therapy combined with drug therapy; while 1 received bronchoscopic therapy combined with external beam radiation therapy (EBRT). 51 patients improved, 1 patient worsened, and 1 died. Meanwhile, 25 patients received drug therapy alone. Among them, 20 patients improved, 4 patients worsened, and 1 died. 2 patients were given EBRT, both of whom improved. 2 patients underwent surgical resection and improved. 27 patients were given symptomatic treatment or only clinical observation, of whom 5 aggravated and 2 died. 66 patients were followed up for more than 3 months. Improvement was found in 2 patients; stabilization was found in 47 patients; exacerbation was found in 9 patients; and death was found in 8 patients (commonly died of lung infection, massive hemorrhage, and respiratory failure) (see Table 4 for details).

\section{Discussion}

Amyloidosis is a group of clinical syndromes caused by deposition of insoluble amyloid fibrils in extracellular matrix, which produces mass effect and cytotoxicity, thereby damaging normal tissue and organ structures and ultimately leading to target organ dysfunction [29]. Amyloidosis may affect various tissues and organs, commonly kidneys, heart, liver, nervous system, and skin, while very rarely it affects trachea and bronchi.

In 1877, Lesser first reported respiratory amyloidosis [30], but so far its specific pathogenesis is unclear, which has been speculated by some scholars to be associated with immunerelated disorders such as plasma cell clone and excessive immunoglobulin secretion [31-33]. TBA is a latent chronic disease. We found through retrospective analysis that the duration of TBA can exceed 30 years and that up to $25 \%$ of patients had TBA duration longer than 5 years. Our data show that the disease is prevalent among elderly people, with a peak onset age of 50-60 years, which is slightly more common in men than in women as well [34]. Clinical manifestations of TBA patients are closely related to the sites and severity of lesions. Common symptoms include cough, shortness of breath, progressive dyspnea, expectoration, and hemoptysis. TBA mainly affects the trachea, main bronchus, lobar bronchi, and segmental bronchi, while it has little impact on small airways and alveoli. Thus, TBA is generally 
TABLE 1: General information of patients.

\begin{tabular}{|c|c|c|}
\hline Feature & $N=109$ & $\%$ \\
\hline \multicolumn{3}{|l|}{ Report time } \\
\hline Years 1981-1999 & $23 / 109$ & 21.1 \\
\hline Years 2000-2015 & $86 / 109$ & 78.9 \\
\hline \multicolumn{3}{|l|}{ Gender } \\
\hline Male & $67 / 109$ & 61.5 \\
\hline Female & $42 / 109$ & 38.5 \\
\hline \multicolumn{3}{|l|}{ Age of onset } \\
\hline$\leq 65$ years & $16 / 109$ & 14.7 \\
\hline$>65$ years & $93 / 109$ & 85.3 \\
\hline \multicolumn{3}{|l|}{ Disease duration $(n=60)$} \\
\hline$\leq 5$ years & $45 / 60$ & 75.0 \\
\hline$>5$ years & $15 / 60$ & 25.0 \\
\hline \multicolumn{3}{|l|}{ Clinical manifestations } \\
\hline Cough & $76 / 109$ & 69.7 \\
\hline Shortness of breath & $67 / 109$ & 61.5 \\
\hline Expectoration & $46 / 109$ & 42.2 \\
\hline Hemoptysis & $33 / 109$ & 30.3 \\
\hline Fever & $17 / 109$ & 15.6 \\
\hline Chest tightness & $10 / 109$ & 9.2 \\
\hline Hoarseness & $7 / 109$ & 6.4 \\
\hline Chest pain & $2 / 109$ & 1.8 \\
\hline Fatigue & $2 / 109$ & 1.8 \\
\hline Misdiagnosis at first hospitalization & $49 / 109$ & 45.0 \\
\hline Chronic bronchitis & $18 / 109$ & 16.5 \\
\hline Endobronchial tuberculosis & $8 / 109$ & 7.3 \\
\hline Lung tumor & $7 / 109$ & 6.4 \\
\hline Bronchial asthma & $7 / 109$ & 6.4 \\
\hline Pulmonary infection & $6 / 109$ & 5.5 \\
\hline COPD & $2 / 109$ & 1.8 \\
\hline Bronchiectasis & $1 / 109$ & 0.9 \\
\hline
\end{tabular}

COPD: chronic obstructive pulmonary disease.

manifested as varying degrees of obstructive ventilatory dysfunction by lung function test. Chest X-ray manifestations are generally increased, disorderly, or normal lung markings or patchy shadows and atelectasis when accompanied with obstructive pneumonia. Chest CT has certain advantages compared to chest X-ray [35], but for early amyloidosis with no obvious bronchial thickening or stenosis, its diagnostic effect is not very satisfactory. Soussan et al. has reported that FDG PET/CT might be valuable for the evaluation of tracheobronchial amyloidosis metabolic activity and followup [36]. However, it has not been promoted due to the high expenses. As clinical manifestations and imaging features of TBA lack specificity, it is often misdiagnosed as chronic bronchitis, endobronchial tuberculosis, lung tumor, bronchial asthma, bronchiectasis, and so forth. Statistics of data in this paper show that initial misdiagnosis rate of TBA is up to $45 \%$, and for the two patients diagnosed in our hospital,
TABLE 2: Imaging manifestations.

\begin{tabular}{lcc}
\hline Feature & $N=109$ & $\%$ \\
\hline Lung CT $(n=82)$ & & \\
Tracheal stenosis & $47 / 82$ & 57.3 \\
Tracheal wall thickening & $42 / 82$ & 51.2 \\
Wall calcification & $17 / 82$ & 20.7 \\
Patchy shadows & $15 / 82$ & 18.3 \\
Atelectasis & $13 / 82$ & 15.9 \\
Hilar space-occupying lesions & $6 / 82$ & 7.3 \\
Normal & $11 / 82$ & 13.4 \\
Chest X-ray ( $n=59)$ & & \\
Increased, disorderly lung markings & $13 / 59$ & 22.0 \\
Atelectasis & $11 / 59$ & 18.6 \\
Patchy shadows & $8 / 59$ & 13.6 \\
Bronchitis & $5 / 59$ & 8.5 \\
Emphysema & $5 / 59$ & 8.5 \\
Luminal stenosis & $5 / 59$ & 8.5 \\
Enlarged hilar shadows & $4 / 59$ & 6.8 \\
Normal & $16 / 59$ & 27.0 \\
\hline
\end{tabular}

CT: computerized tomography.

TABLE 3: Bronchoscopic and histopathological examinations.

\begin{tabular}{lcc}
\hline Feature & $N=109$ & $\%$ \\
\hline Bronchoscopy $(n=107)$ & & \\
Multiple intraluminal nodules, masses & $64 / 107$ & 59.9 \\
Luminal stenosis, occlusion & $54 / 107$ & 50.5 \\
Bronchial wall thickening & $30 / 107$ & 28.0 \\
Brittle and easily bleeding mucosa & $26 / 107$ & 24.3 \\
Mucosal unevenness & $23 / 107$ & 21.5 \\
Mucosal hyperemia and edema & $22 / 107$ & 20.6 \\
Mucosal paleness & $1 / 107$ & 0.9 \\
Wall rigidity & $1 / 107$ & 0.9 \\
Histopathology ( $n=109)$ & & \\
Submucosal amyloid deposition & $109 / 109$ & 100.0 \\
Brick red amyloid by Congo red staining & $50 / 109$ & 45.9 \\
Yellow-green birefringence of amyloid by & $22 / 109$ & 20.2 \\
polarizing microscopy & & \\
\hline
\end{tabular}

one was misdiagnosed for 20 years, whereas the other was misdiagnosed for as long as 30 years.

Introduction and extensive application of bronchoscopy have shed a new light on the diagnosis and treatment of TBA. Through literature review, we found that among TBA cases included in this study, only 23 were reported before 2000, while the number of cases reported since 2000 increased to 86 cases. For this phenomenon, we believe that improved TBA diagnostic technique is a factor that cannot be ignored aside from clinicians' increased awareness of the disease, especially the development and popularization of bronchoscopic techniques in the past decade, which play a vital role [37]. Bronchoscopy not only allows 
TABLE 4: Treatments and outcomes.

\begin{tabular}{|c|c|c|c|c|}
\hline \multirow{2}{*}{ Feature } & \multirow{2}{*}{$N=109$} & \multicolumn{3}{|c|}{ Outcomes } \\
\hline & & Improvement & Exacerbation & Death \\
\hline Means of bronchoscopic therapy $(n=53)$ & & 51 & 1 & 1 \\
\hline Nd-YAG laser & $19 / 53$ & & & \\
\hline APC & $18 / 53$ & & & \\
\hline Freezing & $15 / 53$ & & & \\
\hline Drug spraying & $12 / 53$ & & & \\
\hline Clamping & $6 / 53$ & & & \\
\hline Resection & $5 / 53$ & & & \\
\hline High-frequency electrotome cautery & $4 / 53$ & & & \\
\hline Stent implantation & $2 / 53$ & & & \\
\hline Microwaving & $1 / 53$ & & & \\
\hline Bronchoscopy/other treatments $(n=53)$ & & 51 & 1 & 1 \\
\hline Bronchoscopic therapy alone & $20 / 53$ & & & \\
\hline Combined with drug therapy & $32 / 53$ & & & \\
\hline Combined with EBRT & $1 / 53$ & & & \\
\hline Drug therapy alone $(n=25)$ & $25 / 25$ & 20 & 4 & 1 \\
\hline EBRT/other treatments $(n=2)$ & & 2 & 0 & 0 \\
\hline Combined with glucocorticoids & $1 / 2$ & & & \\
\hline Combined with glucocorticoids + colchicine & $1 / 2$ & & & \\
\hline Surgical resection $(n=2)$ & $2 / 2$ & 2 & 0 & 0 \\
\hline Clinical observation/symptomatic treatment $(n=27)$ & $27 / 27$ & 20 & 5 & 2 \\
\hline Follow-up $\geq 3$ months & $66 / 109$ & 49 & 9 & 8 \\
\hline
\end{tabular}

APC: argon plasma coagulation; EBRT: external beam radiation therapy.

comprehensive and intuitive observation of the sites, types, involving scope and severity of tracheal and bronchial lesions, but also enables condition assessment according to the extent of disease, degree of airway stenosis, and presence or absence of complications such as obstructive pneumonia and airway hemorrhage in patients, based on which prognosis is evaluated. According to a literature report [38], patients with lesions involving the proximal airway and severe middle airway involvement had poor outcomes, most of whom died of progressive aggravated respiratory failure, pulmonary infection, and massive hemorrhage. Depending on the scope of tracheobronchial amyloid deposition and characteristics of lesions, bronchoscopic manifestations of TBA can be classified into three types: (1) multifocal tracheal, bronchial submucosal plaques (the most common type); (2) unifocal nodule or tumor-like mass; and (3) diffuse or infiltrative [39]. TBA has distinct morphological features under bronchoscopy, which are easier to identify. Common manifestations include single or multiple nodules and masses within tracheal and bronchial lumens, localized or diffuse luminal stenosis and occlusion, wall thickening and rigidity, rough or uneven inner luminal wall, mucosal hyperemia and edema, and brittle and easily bleeding mucosa. Narrowband imaging (NBI) bronchoscopy launched in recent years is an emerging bronchoscopic technique, which is mainly used for the diagnosis of early central lung cancer and precancerous lesions. Serrano-Fernández et al. [40] found that if three vascular signs, capillary loops, formation of complex vascular networks, and sudden interruption of large blood vessels, were observed under NBI bronchoscopy, TBA should be highly possible, which could be differentiated from bronchopulmonary cancer as well. Since NBI bronchoscopy is rather sensitive to early TBA and can detect minute lesions, it is conducive to early diagnosis and intervention and helps improve patient prognosis.

Histopathological biopsy is the gold standard for diagnosis of TBA, while bronchoscopic biopsy is the primary means of confirming TBA diagnosis. Compared to the open lung biopsy, bronchoscopic biopsy has advantages such as small invasion, easy and accurate sampling, and little complications. 107 of 109 patients analyzed in this study were diagnosed by bronchoscopic biopsy, which sufficiently shows the significance of bronchoscopy in diagnosing TBA. Histologically, amyloids under tracheobronchial mucosa were presented as homogeneous, amorphous eosinophilic substance by HE staining, which showed brick red color under light microscope by Congo red staining and yellow-green birefringence under polarizing microscope [41].

At present, major treatments of TBA are drug therapy, surgical resection, EBRT [42], and bronchoscopic interventions, as well as temporary follow-up observation (for asymptomatic patients). Since the mid-1990s, the Boston University Amyloidosis Center has used myeloablative-dose melphalan plus autologous hematopoietic stem-cell transplantation; however, up to $15 \%$ of patients experienced severe complications $[43,44]$. Despite good efficacy of classical chemotherapy melphalan-prednisone (MP regimen) for systemic amyloidosis, its efficacy for primary TBA is uncertain 
[45]. Surgical resection is only suitable for TBA patients in general good condition with localized lesions but has the risk of hemorrhage [46]. Bronchoscopic interventions for treating TBA include Nd-YAG laser [47], high-frequency electrotome cautery, freezing, resection, clamping, APC, microwaving, stent implantation [48], and drug spraying [37], which can be used in combination with other treatments. In the literatures statistically analyzed herein, most of patients who received bronchoscopic interventions got the desired short-term effect [49]. However, data for evaluating long-term efficacy are still lacking, so regular bronchoscopic reexamination can be performed to evaluate the therapeutic effect. Although bronchoscopic interventions can quickly relieve the symptoms, multiple repeated interventions are often required as TBA can relapse.

In summary, when we encounter unexplained chronic cough, progressive dyspnea, and recurrent noninfectious obstructive pneumonia in clinical practice, the possibility of TBA should be considered [50]. This will remind us to further confirm the diagnosis by bronchoscopy or by biopsy, if necessary. Bronchoscopy has important value in the diagnosis, condition assessment, treatment, and efficacy evaluation of TBA.

\section{Competing Interests}

The authors declare that there are no competing interests regarding the publication of this paper.

\section{References}

[1] M. A. Gertz, M. Q. Lacy, A. Dispenzieri, and S. R. Hayman, "Amyloidosis," Best Practice \& Research: Clinical Haematology, vol. 18, no. 4, pp. 709-727, 2005.

[2] A. O’Regan, H. M. Fenlon, J. F. Beamis Jr., M. P. Steele, M. Skinner, and J. L. Berk, "Tracheobronchial amyloidosis: The Boston University experience from 1984 to 1999," Medicine, vol. 79, no. 2, pp. 69-79, 2000.

[3] G. Chatkin, M. Pipkin, J. A. F. Pinto, V. D. Da Silva, and J. M. Chatkin, "Primary tracheobronchial amyloidosis," Jornal Brasileiro de Pneumologia, vol. 34, no. 7, pp. 528-531, 2008.

[4] S. K. Sharma, G. Ahluwalia, A. Ahluwalia, and S. Mukhopadhyay, "Tracheobronchial amyloidosis masquerading as bronchial asthma," The Indian Journal of Chest Diseases \& Allied Sciences, vol. 46, no. 2, pp. 117-119, 2004.

[5] Y. Man, Primary Bronchus Amyloidosis Was Diagnosed by CT: A Case Report and Literature Review, Zhejiang University, Hangzhou, China, 2011.

[6] G. Q. Wang, N. S. Zhong, S. Y. Yao et al., "The high frequency electrotome- fiberobronchoscopy treatment for primary respiratory amyloidosis," Journal of Postgraduates of Medicine, vol. 15, 1987.

[7] R. F. Shi, "Respiratory amyloidosis: one case report," Chinese Journal of Practical Internal Medicine, vol. 140, 2009.

[8] S. Zang, A. S. Sun, and L. Y. Zhang, "Respiratory amyloidosis: one case report," Heilongjiang Medical Journal, vol. 50, 1993.

[9] X. N. Zhang, J. Y. Ma, J. F. Zhou, and Y. Y. Sun, "Respiratory amyloidosis: one case report," Hebei Medical Journal, vol. 392, 1994.
[10] X. N. Luo, S. H. Chen, P. N. Wu, L. S. Chen, and X. H. Song, "Analysis of diagnosis and treatment of 7 cases of respiratory amyloidosis," The Journal of Practical Medicine, pp. 207-208, 2007.

[11] X. D. Mou, Y. Xiong, J. Chen, and J. Chen, "Clinical analysis of 11 cases of respiratory amyloidosis," Chinese Journal of Tuberculosis and Respiratory Diseases, vol. 36, pp. 88-93, 2013.

[12] M. Bai, Y. Shang, and Z. H. Tang, "10 cases of primary tracheal bronchus amyloidosis were treated by fiberobronchoscopy laser," The Journal of Inner Mongolia Nationality University, pp. 574-575, 2004.

[13] C. F. Liu, J. Xu, L. L. Geng, and P. Li, "A case of primary tracheal amyloidosis was treated by fiberobronchoscopy," Chinese Journal of Tuberculosis and Respiratory Diseases, vol. 34, pp. 479480, 2011.

[14] D. F. Zhu and G. S. Rong, “Tracheal bronchus amyloidosis: one case report and literature review," Journal of Clinical Pulmonary Medicine, pp. 2326-2328, 2014.

[15] H. S. Cao, C. S. Niu, and Y. J. Wu, "Extensive tracheal bronchus amyloidosis induced respiratory obstruction during surgery: one case report," Journal of Henan University, vol. 85, 1987.

[16] Y. H. Zhang, K. W. Huang, W. H. Chen, R. Z. Liang, and L. J. Guo, "Clinical analysis of 7 cases of tracheal bronchus amyloidosis," International Journal of Respiration, vol. 30, pp. 1161-1164, 2010.

[17] M. Z. He, C. Cai, S. Y. Li, Y. W. Jiang, Y. Chen, and N. S. Zhong, "Clinical analysis of 13 cases of tracheal bronchus amyloidosis," Chinese Journal of Tuberculosis and Respiratory Diseases, vol. 35, pp. 930-931, 2012.

[18] Q. Qu and X. Wang, “3 Cases of tracheal bronchus amyloidosis," Journal of Baotou Medical College, pp. 380-381, 2000.

[19] H. Y. Wang, "Tracheal bronchus amyloidosis: one case report," Beijing Medical Journal, vol. 34, 2005.

[20] H. Sun, "One case of tracheal bronchus amyloidosis was misdiagnosed with chronic obstructive pulmonary disease," The Journal of Practical Medicine, vol. 3069, 2013.

[21] P. Li, X. W. Feng, and L. Zhao, "Lower respiratory amyloidosis: 3 cases report and literature review," China Journal of Endoscopy, pp. 392-395, 2011.

[22] H. Chen and Y. W. Fu, "Argon knife in combination with fiberobronchoscope treat bronchus amyloidosis," Clinical Misdiagnosis \& Mistherapy, vol. 24, pp. 289-290, 2007.

[23] W. W. Li and S. H. Ren, "One case of tracheal bronchus amyloidosis was treated by chest external radiation therapy," Chinese Journal of Tuberculosis and Respiratory Diseases, vol. 31, pp. 544-545, 2008.

[24] S. H. Ren, "3 Cases of primary lung amyloidosis were treated by chest external radiation therapy," Chinese Medical Journal, vol. 89, pp. 2874-2876, 2009.

[25] L. H. Xu, A. M. Wang, and J. F. Zhang, "The nursing care of one tracheal amyloidosis who was treated by Argon plasma coagulation," Journal of Nursing Science, pp. 36-37, 2013.

[26] X. F. Wang, Y. Q. Wang, and C. E. Yang, "Primary amyloidosis," Journal of Harbin Medical University, vol. 285, 1988.

[27] Q. L. Jia, B. Wang, and Z. L. Liu, "One case of primary amyloidosis," Shanxi Medical Journal, vol. 213, 2008.

[28] J. S. Geng, Z. Z. Yuan, and B. N. Qin, "Primary respiratory amyloidosis: one case report," People’s Military Surgeo 37, 1993.

[29] M. F. Khan and R. H. Falk, "Amyloidosis," Postgraduate Medical Journal, vol. 77, no. 913, pp. 686-693, 2001. 
[30] A. Lesser, "Ein Fall von Enchondroma osteoides mixtum der Lunge mit partieller Amyloidentartung," Archiv für Pathologische Anatomie und Physiologie und für Klinische Medicin, vol. 69, no. 3-4, pp. 404-409, 1877.

[31] G. Merlini and V. Bellotti, "Molecular mechanisms of amyloidosis," New England Journal of Medicine, vol. 349, no. 6, pp. 583596, 2003.

[32] R. A. Kyle and M. A. Gertz, "Primary systemic amyloidosis: clinical and laboratory features in 474 cases," Seminars in Hematology, vol. 32, no. 1, pp. 45-59, 1995.

[33] J. Teng, E. A. Turbat-Herrera, and G. A. Herrera, "Role of translational research advancing the understanding of the pathogenesis of light chain-mediated glomerulopathies," Pathology International, vol. 57, no. 7, pp. 398-412, 2007.

[34] A. D. Wechalekar, H. J. B. Goodman, H. J. Lachmann, M. Offer, P. N. Hawkins, and J. D. Gillmore, "Safety and efficacy of riskadapted cyclophosphamide, thalidomide, and dexamethasone in systemic AL amyloidosis," Blood, vol. 109, no. 2, pp. 457-464, 2007.

[35] A. N. Uddin, D. R. Mansfield, M. W. Farmer, and K. K. Lau, "Primary tracheobronchial amyloidosis associated with tracheobronchomegaly evaluated by novel four-dimensional functional CT,' Respirology Case Reports, vol. 3, no. 4, pp. 151154, 2015.

[36] M. Soussan, M.-J. Ouvrier, G. Pop, J.-L. Galas, A. Neuman, and P. Weinmann, "Tracheobronchial FDG uptake in primary amyloidosis detected by PET/CT," Clinical Nuclear Medicine, vol. 36, no. 8, pp. 723-724, 2011.

[37] J. Berraondo, L. Novella, F. Sanz, R. Lluch, E. De Casimiro, and T. Lloret, "Management of tracheobronchial amyloidosis with therapeutic bronchoscopic techniques," Archivos de Bronconeumologia, vol. 49, no. 5, pp. 207-209, 2013.

[38] J. T. Daniels, J. D. Cury, and J. Diaz, "An unusual cause of postobstructive pneumonia," Chest, vol. 131, no. 3, pp. 930-933, 2007.

[39] C. Ozer, M. Nass Duce, A. Yildiz, F. D. Apaydin, H. Eilmez, and T. Arpaci, "Primary diffuse tracheobrochial amyloidosis: case report," European Journal of Radiology, vol. 44, no. 1, pp. 37-39, 2002.

[40] M. L. Serrano-Fernández, P. Álvarez-Maldonado, G. AristiUrista, A. Valero-Gómez, R. Cicero-Sabido, and C. N.-P. Redondo, "Narrow-band imaging bronchoscopy in tracheobronchial amyloidosis," Journal of Bronchology and Interventional Pulmonology, vol. 21, no. 3, pp. 267-270, 2014.

[41] Y. Y. Ying, D. D. Ma, and W. Xiao, "The value of polarizing microscope in diagnosis of primary tracheal bronchus amyloidosis and literature review," Journal of Shandong University (Medical Edition), pp. 67-69, 2014.

[42] S. Ren and G. Ren, "External beam radiation therapy is safe and effective in treating primary pulmonary amyloidosis," Respiratory Medicine, vol. 106, no. 7, pp. 1063-1069, 2012.

[43] S. Mahmood, G. Palladini, V. Sanchorawala, and A. Wechalekar, "Update on treatment of light chain amyloidosis," Haematologica, vol. 99, no. 2, pp. 209-221, 2014.

[44] V. Sanchorawala, M. Skinner, K. Quillen, K. T. Finn, G. Doros, and D. C. Seldin, "Long-term outcome of patients with AL amyloidosis treated with high-dose melphalan and stem-cell transplantation," Blood, vol. 110, no. 10, pp. 3561-3563, 2007.

[45] M. A. Gertz, "How to manage primary amyloidosis," Leukemia, vol. 26, no. 2, pp. 191-198, 2012.
[46] H. D. Attwood, C. G. Price, and R. J. Riddell, "Primary diffuse tracheobronchial amyloidosis," Thorax, vol. 27, no. 5, pp. 620624, 1972.

[47] H. Inaty, E. Folch, C. Stephen, and A. Majid, “Tracheobronchial amyloidosis in a patient with Sjogren syndrome," Journal of Bronchology and Interventional Pulmonology, vol. 20, no. 3, pp. 261-265, 2013.

[48] A. Fiorelli, M. Accardo, G. Galluccio, and M. Santini, “Tracheobronchial amyloidosis treated by endobronchial laser resection and self expanding Y stent," Archivos de Bronconeumologia, vol. 49, no. 7, pp. 303-305, 2013.

[49] I. Alloubi, M. Thumerel, H. Bégueret, J.-M. Baste, J.-F. Velly, and J. Jougon, "Outcomes after bronchoscopic procedures for primary tracheobronchial amyloidosis: retrospective study of 6 cases," Pulmonary Medicine, vol. 2012, Article ID 352719, 4 pages, 2012.

[50] E. Dumoulin, "Primary tracheobronchial amyloidosis: commonly uncommon?" Canadian Respiratory Journal, vol. 21, no. 5, p. 270, 2014. 


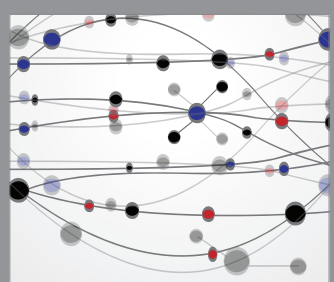

The Scientific World Journal
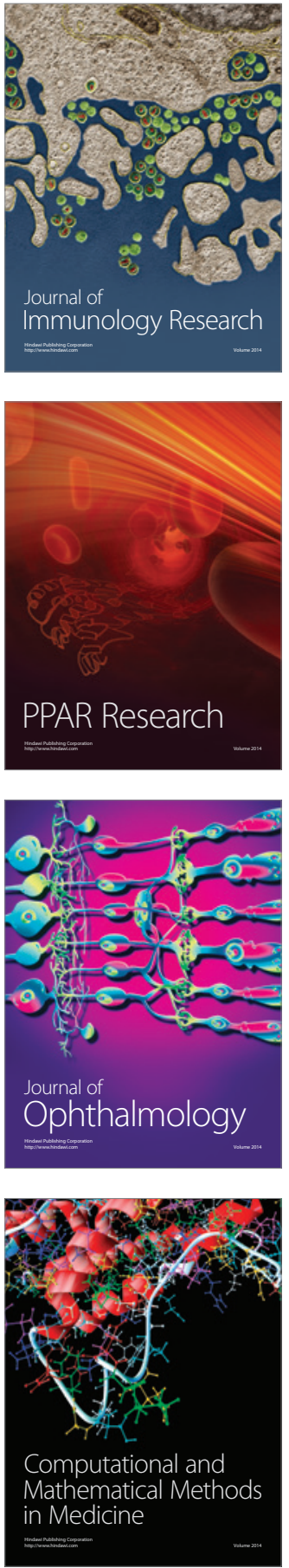

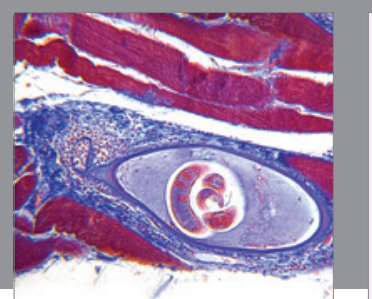

Gastroenterology Research and Practice
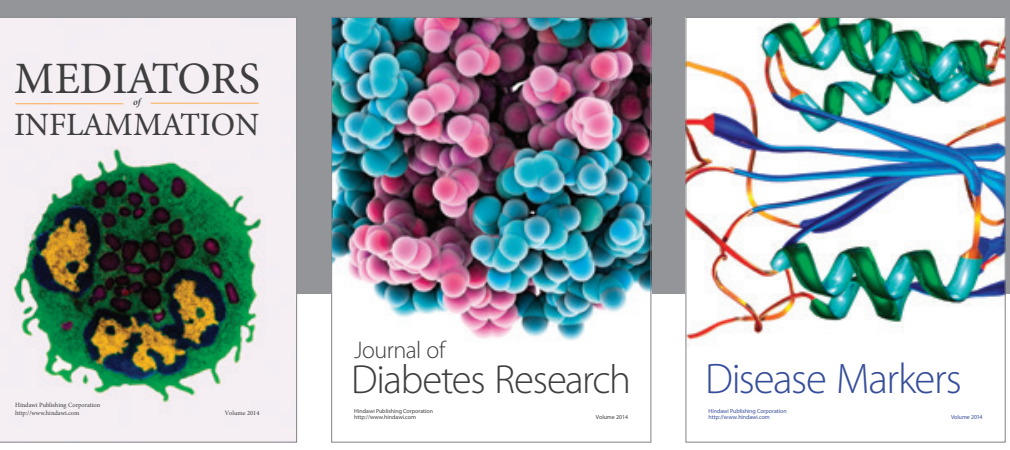

Disease Markers

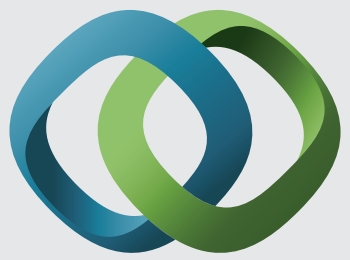

\section{Hindawi}

Submit your manuscripts at

https://www.hindawi.com
